\title{
COMPARISON OF SURFACE RADIATION DOSE TO THE GONADS BY RADIOGRAPHIC EXAMINATION OF THE LUMBAR SPINE USING COMPUTED RADIOGRAPHY AND DIRECT DIGITAL RADIOGRAPHY
}

\author{
PRIYANKA, RAHUL P KOTIAN*, NITIKA C PANAKKAL
}

Department of Medical Imaging Technology, Manipal School of Allied Health Sciences, Manipal University, Manipal, Karnataka, India. Email: kotian.rahu18@gmail.com

Received: 30 June 2017, Revised and Accepted: 11 August 2017

\begin{abstract}
Introduction: Lumbar spine radiography is an inexpensive and quick imaging technique which is often used for the evaluation of bony structures and initial screening examination for suspected fractures, congenital spinal defects, malalignment, and degenerative changes of the spine. During lumbar spine radiography, gonads are also irradiated. As gonads are radiosensitive organs, biological damage produced by radiation is closely related to the amount of energy absorbed. Surface radiation dose (SRD) is a measurement of dose received by the skin of the patient where the X-ray beam passes through the patient.
\end{abstract}

Objectives: The objectives are aimed to study the comparison of SRD to the gonads by radiographic examination of the lumbar spine using computed radiography (CR) and direct digital radiography (DR) and to evaluate dose differences according to gender.

Methods: A total of 120 subjects with an equal number of males and females referred for lumbar spine anteroposterior (AP) and lateral was included in the study. Sixty patients had undergone X-ray in CR X-ray unit and 60 in DR X-ray unit. SRD to gonads from a radiographic examination of the lumbar spine was measured in CR and DR using Mult-O-Meter, and obtained value of SRD was in $\mu$ Gy. Statistical analysis was performed using Statistical Package for the Social Sciences. Data were represented as a median and interquartile range. Mann-Whitney U-test was used for the comparison of SRD to gonads. Two-way analysis of variance (ANOVA) test was used to find out the statistically significant difference in SRD to the gonads according to gender from radiography of lumbar spine taken using CR and direct DR.

Results: There was a statistically significant difference in SRD to gonads from radiography of lumbar spine AP and lateral taken using CR and direct DR $(\mathrm{p}<0.001)$. There was no statistically significant difference in SRD to the gonads in males and females from radiography of lumbar spine AP ( $\mathrm{p}=0.577)$ and lateral $(p=0.164)$ taken using CR and direct DR.

Conclusion: It was found that SRD to gonads from lumbar spine AP was 54\% lower in direct DR and SRD to gonads from lumbar spine lateral was $68 \%$ lower in direct DR than CR.

Keywords: Surface radiation dose, Computed radiography, Direct digital radiography, Lumbar spine.

(c) 2017 The Authors. Published by Innovare Academic Sciences Pvt Ltd. This is an open access article under the CC BY license (http://creativecommons. org/licenses/by/4. 0/) DOI: http://dx.doi.org/10.22159/ajpcr.2017.v10i12.21029

\section{INTRODUCTION}

Radiography is often the first line of choice in diagnosis due to its speed, availability, and lower costs compared to other imaging modalities. The lumbar spine radiography is an inexpensive and quick imaging technique which is often used for the evaluation of bony structures and initial screening examination for suspected fractures, congenital spinal defects, malalignment, and degenerative changes of the spine. At present, radiography of lumbar spine is frequently taken in patients with low back pain to evaluate degenerative changes of the spine such as osteoporosis [1,2]. Radiographic examination of the lumbar spine commonly includes anteroposterior (AP) and lateral view. With the increased advantage of direct digital imaging systems for medical diagnostics, digital imaging is necessary in the health care [3].

Radiographic examinations are associated with both benefits and risk. Radiotherapy is one of the effective methods of cancer therapy [4]. Gonads are radiosensitive organs. Radiation can impair oocyte function, leading to impaired or non-fertility. Radiation dose required to have these effect decreases with age due to falling total oocyte numbers. Similarly, radiation exposure to the testes can result in temporary or permanent azoospermia. Permanent sterility occurs after gonads have received 2.5-3.5 Gy [5].
Surface radiation dose (SRD) is a measurement of dose received by the skin of the patient, where the X-ray beam passes through the patient. SRD can be measured using thermoluminescent dosimeter (TLD), pocket dosimeter, or Mult-O-Meter [6].

In this study, SRD to gonads was measured in computed radiography (CR) and direct digital radiography (DR) during radiographic examination of lumbar spine using Mult-O-Meter, and obtained value of SRD was in $\mu$ Gy.

\section{METHODS}

The study was a cross-sectional study, conducted in the Radio Diagnosis and Imaging Department at Kasturba Hospital, Manipal, during April 2016 to March 2017. The sample size was obtained using the formula for the estimation of mean with 5\% level of significance. About 120 subjects with equal males and females referred for AP, and lateral X-ray of the lumbar spine was included in the study. The study approval was acquired by the Institutional Research Committee, School of Allied Health Sciences, and Institutional Ethics Committee, Kasturba Hospital, Manipal. Informed consents were obtained from all the study participants. Subjects with age ranging from 18 to 50 years and body mass index (BMI): 18.5-24.9 were included in the study. Individuals with a history of trauma were excluded from this study. 
Sixty subjects had undergone X-ray in CR-Fujifilm, and 60 subjects had undergone X-ray in DR-Philips Medical Systems digital diagnostic TH. Exposure factors set in direct DR unit was based on automatic exposure control (AEC), whereas manual factors were set in the CR unit ranging from 65 to $75 \mathrm{kVp}$ and 100-125 mAs. For measuring the SRD to gonads from radiographic examination of the lumbar spine in AP and lateral view, a Mult-0-Meter was placed on the surface of the patient close to the pelvic region. Mult-O-Meter consists of a ionization chamber and small external detector that measures $\mathrm{kVp}$, dose, dose rate, and time simultaneously. The obtained value of SRD was in $\mu \mathrm{Gy}$. The effective dose can be calculated from the SRD using the following formula:

$\mathrm{E}=\mathrm{k} \times \mathrm{SRD}$

- $\quad \mathrm{E}=$ Effective dose

- $\mathrm{k}=$ Conversion factor

- $\quad \mathrm{SRD}=$ Surface radiation dose

As per the National Radiological Protection Board (NRPB)-SR262 Monte Carlo data [7], conversion factors used were 0.109 and 0.018 for lumbar spine AP and lumbar spine lateral, respectively.

\section{Statistical analysis}

Statistical analysis was performed using the Statistical Package for the Social Sciences version 22.0. As the SRD to the gonads from radiographic examination of the lumbar spine in AP and lateral view using CR and direct DR was not normally distributed, data were summarized as a median and interquartile range.

Mann-Whitney U-test was used for the comparison of SRD to gonads. Two-way ANOVA test was used to find out the statistically significant difference in SRD to gonads according to gender from radiography of lumbar spine taken using CR and direct DR. Statistical significance was considered at $\mathrm{p}<0.05$.

\section{RESULTS}

A total of 120 subjects with an equal number of males and females referred for lumbar spine AP and lateral was included in the study with age ranging from 18 to 50 years and BMI: 18.5-24.9. From the 120 subjects, 60 subjects had undergone X-ray in CR X-ray unit, and 60 subjects had undergone X-ray in DR X-ray unit.

Median SRD and interquartile range to gonads from lumbar spine AP in CR and direct DR were evaluated. Fig. 1 shows that median SRD to gonads from lumbar spine AP in CR was found to be $271.25 \mu \mathrm{Gy}$ with lower quartile (Q1) of $242.7 \mu \mathrm{Gy}$ and upper quartile of (Q3) $321.65 \mu \mathrm{Gy}$. In DR, the median SRD to gonads was found to be $77.27 \mu \mathrm{Gy}$ with lower quartile (Q1) of $42.51 \mu \mathrm{Gy}$ and upper quartile (Q3) of $112.75 \mu \mathrm{Gy}$.

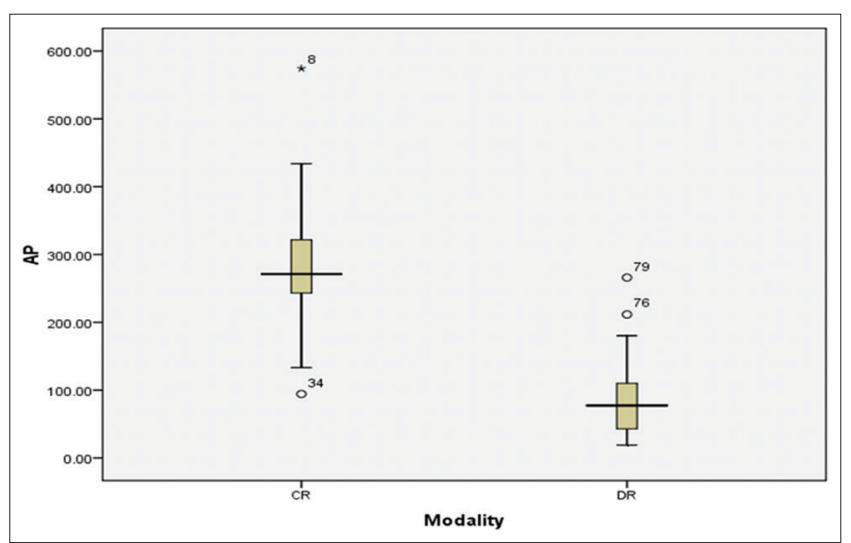

Fig. 1: Box plots representing median surface radiation dose for lumbar spine anteroposterior for computed radiography and direct digital radiography
Mann-Whitney U-test was performed to compare median SRD for lumbar spine AP using CR and direct DR. The test statistic for a Mann-Whitney U-test was 82 , with a $\mathrm{p}<0.001$.Therefore, the results showed that there is a significant difference in median SRD to gonads from lumbar spine AP taken using CR and direct DR.

Median SRD and interquartile range to gonads from of lumbar spine lateral in CR and direct DR were evaluated. Fig. 2 shows that median SRD to gonads from lumbar spine lateral in CR was found to be $432.45 \mu$ Gy with lower quartile (Q1) of $310.20 \mu \mathrm{Gy}$ and upper quartile (Q3) of $660.45 \mu \mathrm{Gy}$. In DR, the median SRD to gonad was found to be $87.98 \mu \mathrm{Gy}$ with lower quartile (Q1) of $62.15 \mu \mathrm{Gy}$ and upper quartile (Q3) of $126.19 \mu \mathrm{Gy}$.

Mann-Whitney U-test was performed to compare median SRD between two groups, namely, lumbar spine lateral taken using CR and direct DR. The test statistic for a Mann-Whitney U-test is 71 , with a $\mathrm{p}<0.001$. The results showed a significant difference in median SRD to gonads from lumbar spine lateral taken using CR and direct DR.

Effective dose was calculated from SRD using a conversion factor. Table 1 shows that mean effective dose to gonads from lumbar spine AP and lateral in CR and DR.

The mean SRD to gonads in males and females from a radiographic examination of lumbar spine AP with $\mathrm{CR}$ and direct DR was also evaluated. SRD to gonads from lumbar spine AP in males was found to be $282.29 \pm 81.92 \mu \mathrm{Gy}$ in CR and $75.24 \pm 43.40$ in DR. The mean SRD to gonads from lumbar spine AP in females was found to be 281.97 $\mu \mathrm{Gy} \pm 83.17$ in CR and $91.05 \mu \mathrm{Gy} \pm 58.11$ in DR as shown in Fig. 3 .

Statistical analysis was performed using two-way ANOVA test. The test revealed that there was a significant difference between $C R$ and direct DR $(p<0.001)$, but there is no significant difference in gender $(p=0.577)$.

Mean SRD to gonads in males and females from radiographic examination of lumbar spine lateral with CR and direct DR was also evaluated. Mean SRD to gonads from lumbar spine lateral in males was

Table 1: Conversion factor and mean effective dose to gonads from lumbar spine AP and lateral taken using CR and DR

\begin{tabular}{llll}
\hline Projections & $\begin{array}{l}\text { Conversion } \\
\text { factor }\end{array}$ & $\begin{array}{l}\text { Effective } \\
\text { dose in } \\
\text { CR }(\boldsymbol{\mu S v})\end{array}$ & $\begin{array}{l}\text { Effective } \\
\text { dose in } \\
\text { DR }(\boldsymbol{\mu S v})\end{array}$ \\
\hline Lumbar spine AP & 0.109 & $30.73 \pm 8.94$ & $9.06 \pm 5.60$ \\
Lumbar spine lateral & 0.018 & $8.38 \pm 3.35$ & $1.83 \pm 4.08$ \\
\hline
\end{tabular}

CR: Computed radiography, DR: Digital radiography, AP: Anteroposterior

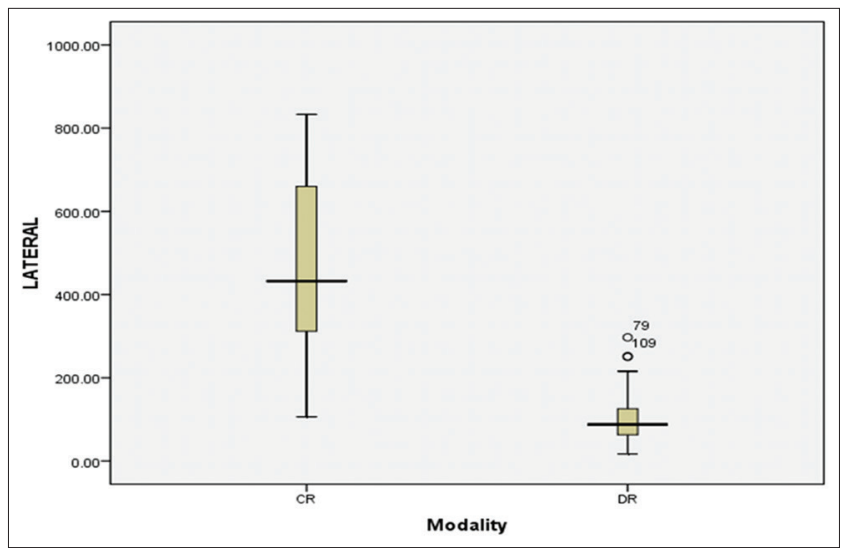

Fig. 2: Box plots representing median surface radiation dose for lumbar spine lateral for computed radiography and direct digital radiography 
Table 2: Mean SRD and percentage differences between the SRD from radiography of lumbar spine using CR and DDR

\begin{tabular}{|c|c|c|c|c|}
\hline Studies & Radiography of lumbar spine & Mean SRD in CR & Mean SRD in DR & DDR versus CR \\
\hline \multirow[t]{2}{*}{ Compagnone et al. } & Lumbar spine AP & 2.54 mGy & 1.16 mGy & $-54 \%$ \\
\hline & Lumbar spine lateral & 5.39 mGy & 1.72 mGy & $-68 \%$ \\
\hline Panicker et al. & Lumbar spine lateral & $19.15 \mathrm{mSv}$ & $11.01 \mathrm{mSv}$ & $-42 \%$ \\
\hline \multirow[t]{2}{*}{ Present study } & Lumbar spine AP & $282.13 \mu \mathrm{Gy}$ & $83.14 \mu \mathrm{Gy}$ & $-70 \%$ \\
\hline & Lumbar spine lateral & $465.56 \mu \mathrm{Gy}$ & $102.11 \mu \mathrm{Gy}$ & $-78 \%$ \\
\hline
\end{tabular}

SRD: Surface radiation dose, CR: Computed radiography, DR: Digital radiography, DDR: Direct digital radiography, AP: Anteroposterior

Table 3: Mean effective dose from radiography of lumbar spine in the present work compared with various data established reported in literature

\begin{tabular}{lll}
\hline Studies & $\begin{array}{l}\text { Radiography of } \\
\text { lumbar spine }\end{array}$ & Mean effective dose (mSv) \\
\hline Gyekye et al. & AP & 10.4 \\
& Lateral & 8.6 \\
Suliman et al. & AP & 34.9 \\
& Lateral & 15.3 \\
Present study & AP & 19.90 \\
& Lateral & 5.10 \\
\hline
\end{tabular}

AP: Anteroposterior

found to be $506.69 \mu \mathrm{Gy} \pm 196.06$ in CR and $96.02 \mu \mathrm{Gy} \pm 56.33$ in DR. Mean SRD to gonads from lumbar spine lateral in females was found to be 424.43 $\mu \mathrm{Gy} \pm 187.03281$ in CR and $108.19 \mu \mathrm{Gy} \pm 58.58$ in DR as shown in Fig. 4.

Statistical analysis was performed using two-way ANOVA test. The test revealed that there was a significant difference between $C R$ and direct DR $(p<0.001)$, but there is no significant difference in gender $(p=0.164)$.

\section{DISCUSSION}

The present study compares the SRD received by the gonads during the radiographic examination of the lumbar spine in CR and direct DR and evaluates dose differences according to gender.

In a study conducted by Compagnone et al., SRD was calculated by the mathematical model using outputs measured by ionization chamber [8]. However, in the present study, the SRD to gonads was measured using Mult-O-Meter, and obtained value of SRD was in $\mu \mathrm{Gy}$. There are various methods to estimate SRD. In a pilot study done by Panicker et al., SRD to the patient undergoing lumbar spine lateral radiography in conventional X-ray unit and the digital X-ray unit was measured using TLD, and obtained value of electrostatic discharge (ESD) was in mSv [9].

In this present study, it was found that SRD to gonads from lumbar spine AP was 70\% lower in DR than CR, whereas SRD to gonads from lumbar spine lateral was 78\% lower in DR than CR. However, the present study compares only CR and DR, a study performed by Compasgnone et al. compares CR, DR, and film-screen systems and also calculated the effective dose. The effective dose in dual delayed-release (DDR) was $29 \%$ lower compared to screen film radiography and $43 \%$ lower in DDR compared to CR. Another study done by Panicker et al. found that ESD from radiography of lumbar spine lateral in DR was $42 \%$ lower than CR X-ray unit (Table 2).

The reason for a lower SRD to gonads in direct DR might be due to various factors such as the greater dynamic range, higher efficiency in signal conversion, and usage of higher $\mathrm{kVp}$ and the lower mAs within the diagnostic range that adequately exposes the image receptor to give a diagnostic quality image. Another possible reason for lower SRD to gonads in DR could be due to the use of AEC during lumbar spine radiography, whereas in $\mathrm{CR}$, exposure factors are set manually by radiographer.

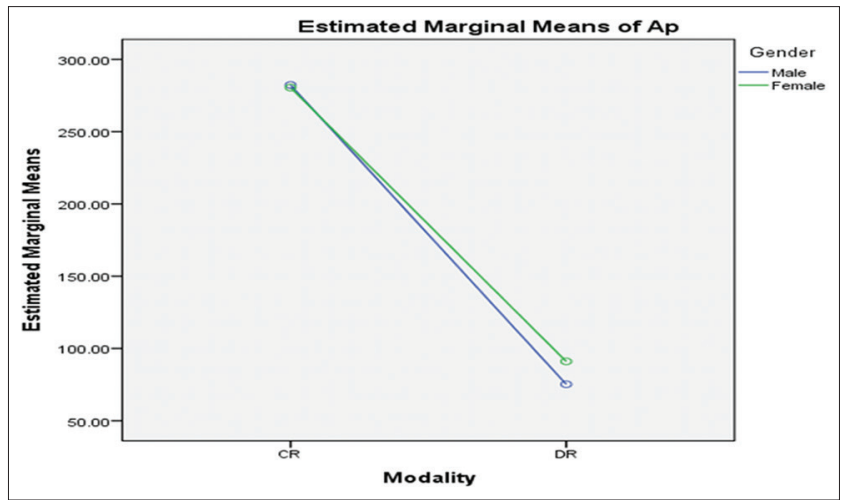

Fig. 3: Profile plots representing mean surface radiation dose for males and females in lumbar spine anteroposterior

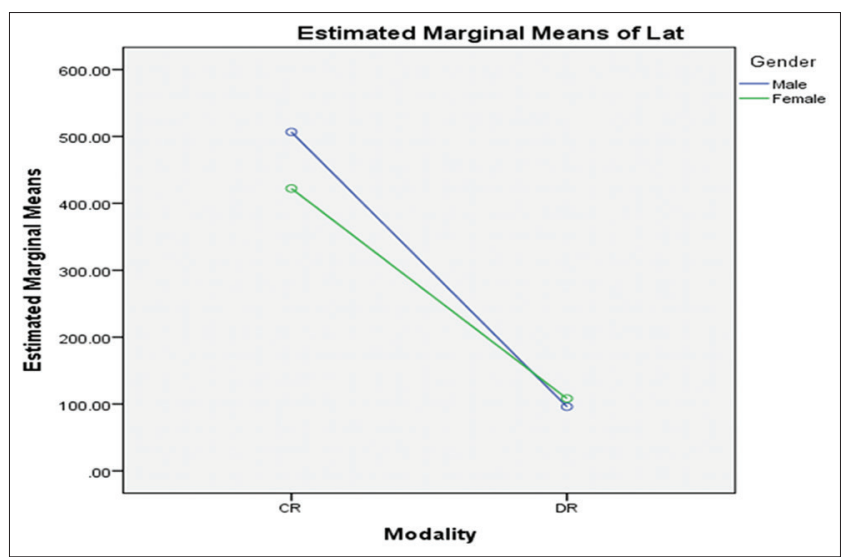

Fig. 4: Profile plots representing mean surface radiation dose for males and females in lumbar spine lateral

The present study also estimated the mean effective dose during lumbar spine radiography in CR and DR using NRPB conversion factors. The mean effective dose to gonads in CR and DR from lumbar spine radiography was found to be $19.56 \mu \mathrm{Sv}$ and $5.45 \mu \mathrm{Sv}$, respectively. A study conducted by Compasgnone et al. showed an effective dose of $0.476 \mathrm{mSv}$ and $0.179 \mathrm{mSv}$ in CR and DR, respectively, for lumbar spine radiography [8]. They used a similar method to calculate the effective dose from SRD using NRPB conversion factors. It was found effective dose of lumbar spine AP was about twice that of lumbar spine lateral which is similar to the present study (Table 3).

A study conducted by Toosi et al. reported that dose to gonads from radiography of lumbar spine using CR for the females was found much higher than for males [10]. The present study also evaluated the mean SRD to gonads in both the genders. It was found that there is no statistically significant difference in SRD to gonads according to gender. However, mean SRD to gonads from radiographic examination of the lumbar spine in AP and lateral using DR was lower than CR in both males and females. 


\section{Limitations and recommendations}

There are limited studies reported to evaluate SRD during an X-ray of the lumbar spine in CR and DR $[8,11,12]$. As a result, SRD and effective dose to the gonads in the present work could not be compared with state and national diagnostic reference levels. Therefore, further studies can be conducted to set up the state and national diagnostic reference levels of SRD and effective dose.

\section{CONCLUSION}

The mean SRD to gonads from lumbar spine AP was found to be $282.13 \mu \mathrm{Gy}$ in CR and 83.14 $\mu$ Gy in direct DR. The mean SRD to gonads from lumbar spine lateral was found to be $465.56 \mu \mathrm{Gy}$ in CR and 102.11 $\mu \mathrm{Gy}$ in direct DR.

It was found that SRD to gonads from lumbar spine AP was $54 \%$ lower in direct DR and SRD to gonads from lumbar spine lateral was $68 \%$ lower in direct DR than CR.

\section{ACKNOWLEDGMENTS}

The authors are very grateful toward all the participants who took part in this study and for the contributions from the Department of Medical Imaging Technology, School of Allied Health Sciences, Manipal University, and the Department of Radio Diagnosis and Imaging, Kasturba Medical College, Manipal, Manipal University.

\section{REFERENCES}

1. Maher C, Underwood M, Buchbinder R. Non-specific low back pain. Lancet 2017;389(10070):736-47.

2. Tripathy A, Adiga S, Shah H, Shanbhag TV, Kumar MD. A retrospective study of clinical profile and drug prescribing pattern in osteoporosis in a tertiary care hospital. Int J Pharm Pharm Sci 2015;7(10):390-3.

3. Oborska-Kumaszynska D, Wisniewska-Kubka S. Analog and digital systems of imaging in roentgenodiagnostics. Pol J Radiol 2010;75(2):73-81

4. Gupta M, Dahiya J, Marwaha RK, Dureja H. Therapies in cancer treatment: An Overview. Int J Pharm Pharm Sci 2015;7(4):1-9.

5. Ogilvy-Stuart AL, Shalet SM. Effect of radiation on the human reproductive system. Environ Health Perspect 1993;101 Suppl 2:109-6.

6. Parry RA, Glaze SA, Archer BR. The AAPM/RSNA physics tutorial for residents. Typical patient radiation doses in diagnostic radiology. Radiographics 1999;19(5):1289-302.

7. Suliman II, Habbani FI. Effective dose Calculations in Conventional Diagnostic X-Ray Examinations for Patients in Major Sudanese Hospitals. World Congress on Medical Physics and Biomedical Engineering; 2006. p. 208791.

8. Compagnone G, Baleni CC, Pagan L, Calzolaio FL, Barozzi L, Bergamini C. Comparison of radiation doses to patients undergoing standard radiographic examinations with conventional screen-film radiography, computed radiography and direct digital radiography. Br J Radiol 2006;79(947):899-904.

9. Panicker TM, Angelina JT, Korath MK, Mohandas K, Jagadeesan K, Jagadeesan K. Entrance skin dose estimation in X-ray Lumbar Spine lateral procedure : Conventional Vs Digital X-ray units . Pilot Study 2013;26(4):219-20.

10. Toosi MB, Nazery M, Zare H. Application of dose-area product meter to measure organ and effective dose in diagnostic radiology at two hospitals, Mashhad, Iran. In: $11^{\text {th }}$ International Congress of the International Radiation Protection Association, Madrid; 2004. p. 1-10.

11. Gyekye PK, Simon A, Geoffrey ER, Johnson Y, Stephen I, Engmann CK, et al. Radiation dose estimation of patients undergoing lumbar spine radiography. J Med Phys 2013;38(4):185-8.

12. Kotian RP, Priyanka, Panakkal NC. A pilot study to compare surface radiation dose to the gonads by radiographic examination of the lumbar spine using computed radiography and direct digital radiography. Int J Pharm Biol Sci 2016;7(3):499-502 